\title{
Prediction of pathologic prognostic factors in patients with lung adenocarcinomas: comparison of thin-section computed tomography and positron emission tomography/computed tomography
}

\author{
Shingo Iwano ${ }^{1 *}$, Mariko Kishimoto ${ }^{1}$, Shinji Ito ${ }^{1}$, Katsuhiko Kato ${ }^{2}$, Rintaro Ito ${ }^{1}$ and Shinji Naganawa ${ }^{1}$
}

\begin{abstract}
Background: The ratio of the maximum diameter of consolidation to the maximum tumor diameter ( $\mathrm{C} / \mathrm{T}$ ratio) on thin-section computed tomography (TSCT) and the maximum standardized uptake value (SUVmax) on ${ }^{18}$ F-fluorodeoxyglucose positron emission tomography/computed tomography (PET/CT) are often used as preoperative independent variables to evaluate the invasiveness of lung adenocarcinoma. We investigated the associations between these independent variables and pathologic invasiveness in pulmonary adenocarcinomas.

Methods: We selected patients with peripheral lung adenocarcinomas, definitively diagnosed by surgical resection, with diameters of $\leq 30 \mathrm{~mm}$ over a 4-year period ending in December 2010. The association between 3 independent variables (tumor size, SUVmax, and C/T ratio) and pathologic prognostic factors was evaluated using logistic analysis.

Results: We evaluated a total of 163 primary lung adenocarcinomas in 148 patients (93 males and 55 females; age range: 34 to 84 years). Using multivariate logistic regression analysis, SUVmax and the C/T ratio were significantly associated with tumor invasiveness (odds ratio $[\mathrm{OR}]=1.227 ; p=0.025$ and $\mathrm{OR}=1.019 ; p=0.008$, respectively). Tumor size was not associated with invasiveness $(O R=1.003 ; p=0.925)$. For solid type adenocarcinomas, only SUVmax was significantly associated with invasiveness ( $\mathrm{OR}=1.558 ; p=0.003)$. For subsolid type adenocarcinomas, only the $\mathrm{C} / \mathrm{T}$ ratio was significantly associated with invasiveness $(\mathrm{OR}=1.030 ; p=0.009)$.

Conclusions: Both the C/T ratio and the SUVmax are significantly correlated with pathologic invasiveness in patients with small lung adenocarcinomas, while there was a difference between the 2 evaluations. Solid type adenocarcinomas with SUVmax values of $\geq 4.4$ and subsolid type adenocarcinomas with $C / T$ ratio $\geq 53 \%$ were so highly invasive.
\end{abstract}

Keywords: Lung neoplasms, Adenocarcinoma, Computed tomography, Positron emission tomography, Fluorodeoxyglucose, Prognostic factor

\section{Background}

Adenocarcinoma is the most common pathological type of peripheral lung cancer. However, even in patients with tumors of $\leq 3 \mathrm{~cm}$ in diameter, the postoperative prognosis varies, with poorly differentiated adenocarcinoma demonstrating a poor prognosis. Therefore, the use of

\footnotetext{
* Correspondence: iwano45@med.nagoya-u.ac.jp

'Nagoya University Graduate School of Medicine, Department of Radiology, 65 Tsurumai-cho, Showa-ku, Nagoya 466-8550, Japan

Full list of author information is available at the end of the article
}

preoperative imaging to determine tumor invasiveness is important, as limited resection (pulmonary segmentation or wide wedge resection) is currently recommended for non-invasive tumors [1-4].

Well-differentiated lung adenocarcinomas are often of a subsolid type, generally indicating a non-invasive nature and a good prognosis. These tumors may show groundglass opacity on thin-section computed tomography (TSCT). On the other hand, poorly differentiated adenocarcinomas are usually of a solid type and have a tendency 
to be invasive, with a poor prognosis. These tumors consist entirely of consolidation on TSCT. This explains the usefulness of the $\mathrm{C} / \mathrm{T}$ ratio, the maximum diameter of consolidation to the maximum tumor diameter on TSCT, in predicting the invasiveness and prognosis in patients with lung adenocarcinoma [1,3-7].

${ }^{18} \mathrm{~F}$-fluorodeoxyglucose (FDG) positron emission tomography/computed tomography (PET/CT) is also essential in the preoperative evaluation of patients with primary lung cancer. The maximum standardized uptake value (SUVmax) of the primary lesion on PET/CT is well correlated with both tumor size and differentiation, with high-grade tumors tending to have a high SUVmax [8-10]. As with the $C / T$ ratio, this value is used to determine tumor invasiveness and predict patient prognosis [11-18].

It is unclear whether the C/T ratio or the SUVmax value is a more suitable preoperative independent variables for predicting tumor invasiveness, or if there are any differences between them. We therefore conducted a retrospective investigation into the association between each of these independent variables, along with tumor size, and the pathologic invasiveness in patients with small $(\leq 3 \mathrm{~cm})$ pulmonary adenocarcinomas. In addition, we determined the most suitable independent variables cutoff levels to predict tumor invasiveness.

\section{Methods}

This retrospective study was approved by the institutional review board (IRB) of our hospital. The IRB waived the need for written informed consent.

\section{Patient selection}

We searched our institution's records from November 2006 to December 2010 and selected patients who had preoperative PET/CT conducted for pulmonary tumors. We then obtained the clinical records, preoperative TSCT images, and postoperative pathological records for these patients. We limited our study to patients with peripheral lung adenocarcinomas of $\leq 30 \mathrm{~mm}$ in diameter that had been definitively diagnosed by pathological examination after surgical resection. We recorded the age, sex, and body weight of all patients, the pathological findings of the primary tumor (maximum diameter, lymph node metastasis, lymphatic permeation, vascular invasion, and pleural involvement) [17-20], and the pathological stage based on the Union for International Cancer Control guidelines, version 7 .

\section{Preoperative TSCT}

All preoperative TSCT examinations were performed using a 64- detector row scanner (Aquilion 64; Toshiba Medical Systems Corp., Tokyo, Japan). All scans were obtained from the lung apex to the diaphragm, during a breath-hold at deep inspiration, using the following parameters: $x$-ray tube voltage, $120 \mathrm{kVp}$; automatic tube-current maximum, 225 mAs; gantry rotation speed, $0.5 \mathrm{sec}$; and beam collimation, $64 \times 0.5 \mathrm{~mm}$. The TSCT images were reconstructed from 0.5 - $\mathrm{mm}$ slices with intervals of $0.5 \mathrm{~mm}$, using a high spatial-frequency reconstruction algorithm (FC52). No contrast medium was used.

\section{Measurement of consolidation/tumor ratio (C/T ratio)}

Two radiologists independently evaluated the TSCT images using lung-window settings (window level, -600 Hounsfield units; window width, 1,800 Hounsfield units). The viewing monitor belonged to a picture archiving and communication system (RapideyeCore, Toshiba Medical Systems Corp., Tokyo, Japan). The radiologists calculated the $\mathrm{C} / \mathrm{T}$ ratio, defined as the maximum diameter of consolidation over the maximum tumor diameter (Figure 1) [1,3-7]. The consolidation component was defined as an area of increased opacification that completely obscured the underlying vascular markings. Ground-glass opacity was defined as an area of a slight, homogenous increase in density that did not obscure the underlying vascular markings. Areas of ground-glass opacity often present as lepidic-predominant adenocarcinoma, typified by lepidic growth along alveoli, without invasive areas. Previous studies have shown that $\mathrm{C} / \mathrm{T}$ ratio is related to aggressiveness and and prognosis in patients with lung adenocarcinoma. The mean value of the 2 observers for each tumor was used for the study analyses to reduce measuring error. We classified tumors into "solid type", tumors containing no ground-glass opacities $(\mathrm{C} / \mathrm{T}$ ratio $=100 \%)$, and "subsolid type", tumors containing any ground-glass opacity $(\mathrm{C} / \mathrm{T}$ ratio $<100 \%)$.

\section{Preoperative PET/CT}

All preoperative $\mathrm{PET} / \mathrm{CT}$ examinations were made using the Biograph 16 scanner (Siemens Medical Solutions, Erlangen, Germany). Patients were required to fast for at least 6 hours prior to imaging, and blood glucose levels were measured immediately prior to injecting FDG. Patients with blood glucose levels of $>150 \mathrm{mg} / \mathrm{dL}$ were excluded from the study $[3,17]$. The FDG dose was determined by body weight, using either $3.7 \mathrm{MBq} / \mathrm{kg}$ (for patients weighing $<60 \mathrm{~kg}$ ) or $4.07 \mathrm{MBq} / \mathrm{kg}$ (for patients weighing $\geq 60 \mathrm{~kg}$ ). Fifty minutes after the intravenous injection of FDG, emission scans were acquired of the area between the proximal femora and the base of the skull; the 3-dimensional mode was used, with $1.7 \mathrm{~min}$ at each bed position. Breath-holding and respiratory gating were not used. PET images were reconstructed using the ordered-subset expectation maximization (OSEM) algorithm with 2 iterations and 16 subsets, incorporating a CT-based transmission map. No contrast medium was used for transmission CT according to the protocol of our institution. 


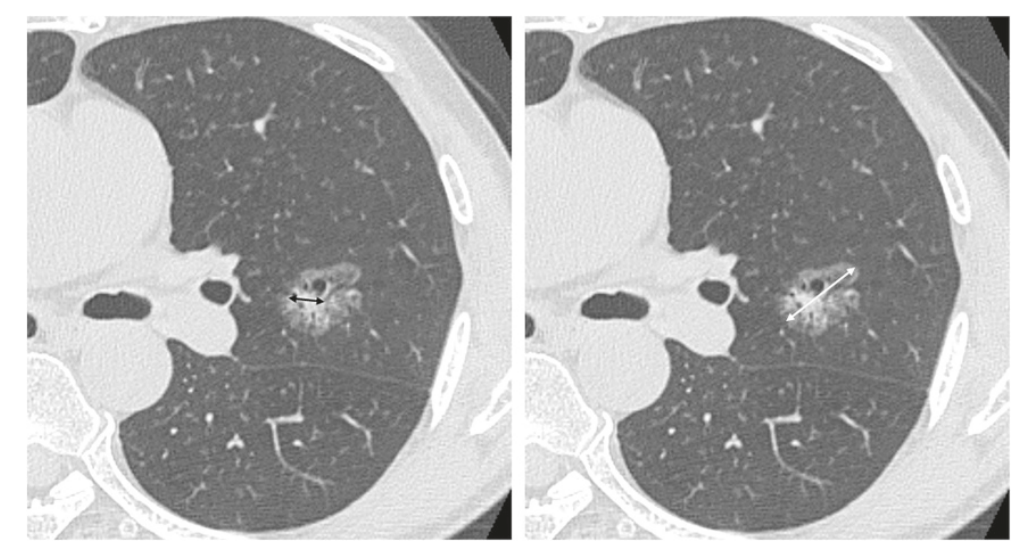

Figure 1 Example of the consolidation/tumor (C/T) ratio measurement on thin-section computed tomography. $C / T$ ratio $(\%)=$ maximum diameter of consolidation (black arrow)/maximum diameter of tumor (white arrow) $\times 100$.

\section{Measurement of maximum standardized uptake value (SUVmax)}

The images were transferred to an e.soft Turbo-V workstation (Siemens Medical Solutions) where fusion PET/ $\mathrm{CT}$ images were reconstructed. Regions of interest (ROIs) were placed around the pulmonary lesions; the maximum SUV value at a given ROI was defined as the SUVmax.

$$
\begin{aligned}
\mathrm{SUV}= & \text { ROI activity }(\mathrm{MBq} / \mathrm{mL}) \times \text { body weight }(\mathrm{g}) \\
& / \text { injected FDG activity }(\mathrm{MBq})
\end{aligned}
$$

The SUVmax represents the maximum glucose metabolic activity of the tumor.

\section{Statistical analysis}

First, the associations between the 3 independent variables (tumor size, SUVmax, and C/T ratio) and the 3 pathologic prognostic factors (pleural involvement, lymphatic/vascular invasion, and lymph node metastasis) were compared using univariate logistic regression analysis. Pearson correlation coefficients between $\mathrm{C} / \mathrm{T}$ ratio versus tumor size, SUVmax versus tumor size, and SUVmax versus $C / T$ ratio were determined. Next, we defined invasive adenocarcinomas as tumors that had at least one invasive pathologic finding (pleural involvement, intratumoral vessel invasion, or lymph node metastasis) and non-invasive adenocarcinomas as tumors that had no invasive pathologic findings [6,7]. Multivariate logistic regression analysis was then used to identify independent predictors of non-invasive or invasive tumor nature, using the 6 independent variables (age, body weight, blood sugar level, tumor size, SUVmax, and $\mathrm{C} / \mathrm{T}$ ratio) for all tumors, then separately for solid type tumors and subsolid type tumors. The mean value for each independent variables was compared, using unpaired Student's t-test, between non-invasive tumors and invasive tumors. Patient characteristics (age, body weight, and blood sugar level) and tumor characteristics (size, SUVmax, and C/T ratio) were compared between the solid and subsolid groups using unpaired Student's t-test, and the frequency of pathological invasiveness was compared between groups using the chi-square test. Receiver operating characteristic (ROC) curve analysis and Youden's index were used to determine a cut-off level that would indicate invasiveness for SUVmax in solid type tumors and for the $\mathrm{C} / \mathrm{T}$ ratio in subsolid type tumors [21]. Finally, recurrence rates within 3 years after surgery were compared using a Chi-square for independence test between non-invasive tumors and invasive tumors.

Excel 2007 (Microsoft Corp., Redmond, WA) and Statistical Package for the Social Sciences, version 21.0 (IBM Corp., Armonk, NY), were used to conduct all statistical analyses. A $p$-value of $<0.05$ was considered significant. Pearson correlation coefficients of $>0.4$ were considered as showing a positive correlation between variables.

\section{Results}

Study's cohort

Figure 2 shows this study's cohort flow chart. A total of 148 patients (93 male and 55 female) were evaluated, with an age range of 34 to 84 years and a median age of 68 years. These 148 patients had a total of 163 primary lung adenocarcinomas (13 patients had 2 simultaneous lesions and 1 patient had 3 lesions). There were a total of 51 lesions (31\%) in the right upper lobe, 11 lesions (7\%) in the right middle lobe, 37 lesions $(23 \%)$ in the right lower lobe, 38 lesions $(23 \%)$ in the left upper lobe, and 26 lesions (16\%) in the left lower lobe. The median maximum lesion diameter was $19.0 \mathrm{~mm}$. The mean \pm standard deviation (SD) $\mathrm{C} / \mathrm{T}$ ratio was $76.3 \pm 33.3 \%$, with a intra-class correlation coefficient between the 2 radiologists of 0.963 (95\% confidential interval $[\mathrm{CI}]=0.950-0.973, p<0.001)$. Eighty-two lesions were of the solid type and 81 were of the subsolid type. The mean \pm SD SUVmax was $2.8 \pm 2.7$. Ninety-two lesions (56\%) had an SUVmax of $<2.5$, and 


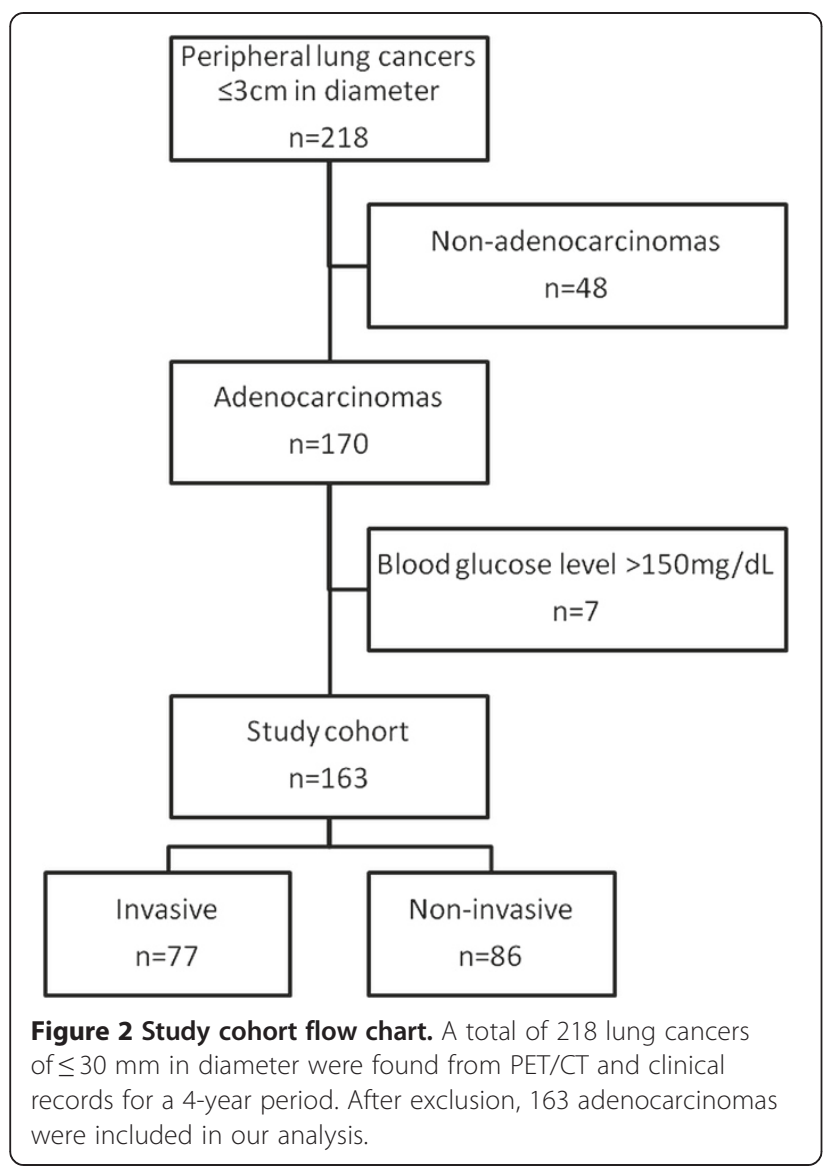

the other 71 lesions (44\%) had an SUVmax of $\geq 2.5$. The histological grade was G1 in 71 tumors, G2 in 76, G3 in 11, and GX (grade cannot be assessed) in 5 tumors. Pleural involvement was observed in 70 lesions, intratumoral vessel invasion (lymphatic and/or vascular invasion) in 24 lesions, and lymph node metastasis in 15 lesions. Sixteen lesions had 2 invasive pathologic findings (pleural involvement, intratumoral vessel invasion, or lymph node metastasis) and 8 lesions had all 3 invasive pathologic findings. Seventy-seven tumors (47\%) were regarded as invasive; these had at least 1 invasive pathologic finding.
The remaining 86 tumors (53\%) were regarded as noninvasive. The pathological stage was stage I in 146 tumors, stage II in 8 , stage III in 8 , and stage IV in 1 tumor. The patient and tumor characteristics are summarized in Table 1.

\section{Association between the independent variables and each pathologic prognostic factor}

Table 2 shows the association between the independent variables and the pathologic prognostic factors using univariate logistic analysis. The tumor size was significantly correlated with pleural involvement and lymph node metastasis ( $p=0.023$ and $p=0.014$, respectively), while size was not correlated with intratumoral vessel invasion $(p=0.158)$. The SUVmax was significantly correlated with intratumoral vessel invasion, pleural involvement, and lymph node metastasis $(p<0.001, p<0.001$, and $p=0.002$, respectively). The $\mathrm{C} / \mathrm{T}$ ratio was significantly correlated with intratumoral vessel invasion and pleural involvement ( $p=0.014$ and $p<0.001$, respectively), but not with lymph node metastasis $(p=0.067)$. There was a positive correlation between SUVmax values and $\mathrm{C} / \mathrm{T}$ ratios (Pearson correlation coefficient $[\mathrm{r}]=0.48, p<0.001)$. On the other hand, there was weak correlation between SUVmax and size $(r=0.36, p<0.001)$; between $\mathrm{C} / \mathrm{T}$ ratio and size $(r=0.20, \mathrm{p}=0.011)$.

\section{Association between the independent variables and the tumor invasiveness}

Table 3 shows the association between independent variables and tumor invasiveness using multivariate logistic regression analysis. The SUVmax and $\mathrm{C} / \mathrm{T}$ ratio were significantly correlated with tumor invasiveness $(p=0.025$ and $p=0.008$, respectively), while tumor size was not correlated with invasiveness $(p=0.925)$. For solid type adenocarcinomas, multivariate logistic analysis demonstrated that only SUVmax was significantly correlated with invasiveness $(p=0.009)$. Conversely, in subsolid type adenocarcinomas, only the $\mathrm{C} / \mathrm{T}$ ratio was significantly correlated with invasiveness $(p=0.003)$. Table 4 shows

Table 1 Patient and tumor characteristics

\begin{tabular}{|c|c|c|c|c|c|c|}
\hline & & Mean & SD & Minimum & IQR & Maximum \\
\hline \multicolumn{7}{|l|}{ Patients } \\
\hline & Age (years) & 66 & 8 & 34 & $62-73$ & 84 \\
\hline & Body weight (kg) & 57 & 11 & 36 & $49-63.5$ & 80 \\
\hline & Blood sugar (mg/dL) & 93 & 14 & 61 & $84-101$ & 141 \\
\hline \multicolumn{7}{|l|}{ Tumors } \\
\hline & Size (mm) & 18.8 & 6.5 & 4 & $13.5-24$ & 30 \\
\hline & SUVmax & 2.8 & 2.7 & ND & $0.9-4.1$ & 14.1 \\
\hline & $\mathrm{C} / \mathrm{T}$ ratio (\%) & 76.3 & 33.3 & 0 & $58.8-100$ & 100 \\
\hline
\end{tabular}


Table 2 Univariate analyses for independent variables and pathologic prognostic factors

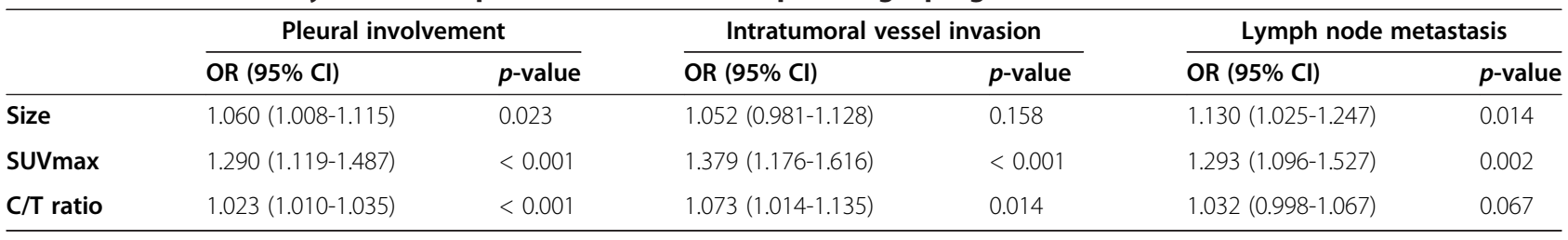

OR: odds ratio; $\mathrm{Cl}$ : confidence interval; SUVmax: maximum standardized uptake value; $\mathrm{C} / \mathrm{T}$ ratio: consolidation diameter/tumor diameter ratio.

the comparison between subsolid type and solid type adenocarcinomas. Solid type tumors had a significantly higher rate of pleural involvement and intratumoral vessel invasion than subsolid type tumors.

The invasive tumors demonstrated a significantly larger size than non-invasive tumors $(p=0.016)$, and higher values for SUVmax and the $\mathrm{C} / \mathrm{T}$ ratio $(p<0.001$ and $p<0.001$, respectively) (Table 5).

\section{A suitable cutoff value for determining invasiveness}

In the analysis of solid type adenocarcinomas, the results of the ROC analysis for SUVmax showed an area under the curve (AUC) of 0.727 (95\% CI $=0.618-0.836)$. A suitable cutoff value for determining invasiveness was estimated to be 4.4. This value yielded a sensitivity, specificity, positive predictive value (PPV), and negative predictive value (NPV) for tumor invasiveness of $55 \%, 88 \%, 84 \%$ and $55 \%$, respectively. In the analysis of subsolid type adenocarcinomas, the results for the $\mathrm{C} / \mathrm{T}$ ratio showed an AUC of 0.736 ( $95 \% \mathrm{CI}=0.618-0.852)$. A suitable cutoff value for determining invasiveness was estimated to be $53 \%$. This value yielded a sensitivity, specificity, PPV, and NPV of $89 \%, 64 \%, 57 \%$ and $92 \%$, respectively.

\section{Postoperative outcomes}

Table 6 shows the postoperative outcomes for 125 patients with solitary lung adenocarcinomas. Twenty patients with simultaneous and metachronous multiple lung cancers were excluded from this analysis. Three patients were also excluded because they changed to another hospital within 3 years after surgery. Invasive adenocarcionomas showed a significantly higher recurrence rate than did non-invasive tumors ( $25 \%$ versus $6 \%, p=0.004)$. Moreover, no local recurrence or distant metastases were found in 16 patients who had limited resections for non-invasive tumors. In contrast, a case of T1N0 invasive adenocarcinoma with intratumoral lymphatic permeation relapsed in a mediastinal lymph node after segmentectomy. This lesion was $13 \mathrm{~mm}$ in diameter, solid type $(\mathrm{C} / \mathrm{T}$ ratio $=$ $100 \%$ ), and had a SUVmax of 4.8 .

Eight of 90 (9\%) patients who had a SUVmax of $<4.4$ relapsed after surgery, while 15 of 35 (43\%) patients who had a SUVmax of $\geq 4.4$ relapsed. No local recurrence or distant metastases were found in 21 patients who had a $\mathrm{C} / \mathrm{T}$ ratio $<53 \%$.

\section{Discussion}

We were able to clarify several points in this study. First, both the $\mathrm{C} / \mathrm{T}$ ratio (on TSCT) and the SUVmax (on $\mathrm{PET} / \mathrm{CT}$ ) are significantly correlated with invasiveness in patients with small adenocarcinomas. Next, an important difference exists between the $\mathrm{C} / \mathrm{T}$ ratio and the SUVmax value: the $C / T$ ratio is useful for predicting invasiveness only in subsolid type adenocarcinomas, while the SUVmax is suitable for solid type tumors. Finally, the cutoff $\mathrm{C} / \mathrm{T}$ ratio for determining invasiveness in subsolid type adenocarcinomas is around 53\%, while the cutoff for SUVmax in solid type adenocarcinomas is around 4.4.

The ability to identify prognostic pathologic factors on preoperative diagnostic imaging is essential to the determination of appropriate therapeutic strategies. Most small pulmonary adenocarcinomas are treated with pulmonary lobectomy, however, there has been a recent call to expand the indications for limited resection (e.g., segmentectomy or wide wedge resection) because of the advantage to the patient in preserving pulmonary

Table 3 Multivariate logistic regression analysis for independent variables and tumor invasiveness

\begin{tabular}{|c|c|c|c|c|c|c|}
\hline & \multicolumn{2}{|c|}{ All $(n=163)$} & \multicolumn{2}{|c|}{ Subsolid type $(n=81)$} & \multicolumn{2}{|c|}{ Solid type $(n=82)$} \\
\hline & OR $(95 \% \mathrm{Cl})$ & $p$-value & OR $(95 \% \mathrm{Cl})$ & $p$-value & OR $(95 \% \mathrm{Cl})$ & $p$-value \\
\hline Age & $1.033(0.988-1.080)$ & 0.158 & $0.982(0.913-1.056)$ & 0.627 & $1.069(1.000-1.142)$ & 0.050 \\
\hline Body weight & $0.996(0.963-1.029)$ & 0.798 & $0.998(0.950-1.048)$ & 0.930 & $1.009(0.957-1.063)$ & 0.746 \\
\hline Blood sugar & $0.982(0.956-1.010)$ & 0.202 & $0.952(0.912-0.994)$ & 0.024 & $1.027(0.979-1.077)$ & 0.279 \\
\hline Size & $1.003(0.944-1.066)$ & 0.925 & $1.032(0.939-1.135)$ & 0.511 & $0.988(0.899-1.086)$ & 0.798 \\
\hline SUVmax & $1.227(1.026-1.469)$ & 0.025 & $0.880(0.636-1.217)$ & 0.439 & $1.558(1.157-2.099)$ & 0.003 \\
\hline $\mathrm{C} / \mathrm{T}$ ratio & $1.019(1.005-1.034)$ & 0.008 & $1.030(1.007-1.053)$ & 0.009 & NA & NA \\
\hline
\end{tabular}

OR: odds ratio; $\mathrm{Cl}$ : confidence interval; SUVmax: maximum standardized uptake value; C/T ratio: consolidation diameter/tumor diameter ratio; NA: not available. 
Table 4 Comparisons between solid type and subsolid type adenocarcinomas

\begin{tabular}{llll}
\hline & Subsolid type $(\mathbf{n}=\mathbf{8 1})$ & Solid type $(\mathbf{n}=\mathbf{8 2})$ & $\mathbf{p}$-value \\
\hline Age (years) & $66 \pm 7$ & $66 \pm 9$ & 0.951 \\
Body weight $(\mathbf{k g})$ & $56 \pm 10$ & $59 \pm 10$ & 0.052 \\
Blood sugar (mg/dL) & $94 \pm 16$ & $92 \pm 11$ & 0.359 \\
Size $(\mathbf{m m})$ & $19.0 \pm 6.7$ & $18.6 \pm 6.2$ & 0.737 \\
SUVmax & $1.7 \pm 2.0$ & $3.8 \pm 2.9$ & $<0.001$ \\
C/T ratio (\%) & $52.3 \pm 33.1$ & $100.0 \pm 0.0$ & $<0.001$ \\
Pleural involvement $(\mathbf{n})$ & $27(33 \%)$ & $43(52 \%)$ & 0.014 \\
Intratumor vessel invasion $(\mathbf{n})$ & $4(5 \%)$ & $20(24 \%)$ & $<0.001$ \\
Lymph node metastasis $(\mathbf{n})$ & $6(7 \%)$ & $9(11 \%)$ & 0.431 \\
Invasive tumor $(\mathbf{n})$ & $28(35 \%)$ & $49(60 \%)$ & 0.001 \\
\hline
\end{tabular}

SUVmax: maximum standardized uptake value; $\mathrm{C} / \mathrm{T}$ ratio: consolidation diameter/tumor diameter ratio.

function. Unfortunately, limited resection carries the risk of locoregional recurrence due to the possibility of inadequate surgical margins and incomplete lymph-node clearance $[1,22-24]$. Therefore, to reduce risk to patients, it is very important to be able to determine preoperatively which tumors are likely to be non-invasive [2-4]. In the present study, a case of adenocarcinoma with a high $\mathrm{C} / \mathrm{T}$ ratio and a high SUVmax value relapsed after segmentectomy. Our results demonstrate that both TSCT and PET/CT findings may be useful in deciding which patients are better suited for limited resection.

We found that the SUVmax was significantly correlated with all pathologic prognostic factors; in other words, patients with higher SUVmax values have more highly invasive tumors. The $\mathrm{C} / \mathrm{T}$ ratio was also significantly correlated with pleural involvement and intratumoral vessel invasion, but not with lymph node metastasis. A higher $\mathrm{C} / \mathrm{T}$ ratio indicates that the tumor is associated with a greater amount of consolidation, and these tumors are more highly invasive. The reason that lymph node metastasis did not show an association with the $\mathrm{C} / \mathrm{T}$ ratio in this study may have been because the absolute number of lymph node metastases was small $(n=15)$. In the present study, we selected lung adenocarcinomas of $\leq 3 \mathrm{~cm}$ in diameter, that is, T1a-b in the TNM staging system by reference to the study of Asamura et al.
[4]. They reported a $\mathrm{C} / \mathrm{T}$ ratio 0.25 or less in $\mathrm{cT} 1 \mathrm{a}$ $(\leq 2 \mathrm{~cm})$ and 0.05 in $\mathrm{cT} 1 \mathrm{a}-\mathrm{b}(\leq 3 \mathrm{~cm})$ were both able to define a homogenous group of patients with an excellent prognosis. In our study, tumor size is correlated with pleural involvement and lymph node metastasis, but not with intratumoral vessel invasion. Multivariate analysis showed that both the $\mathrm{C} / \mathrm{T}$ ratio and the SUVmax value are independent and significant variables for predicting the invasiveness of lung adenocarcinoma, but tumor size is not significant. The $\mathrm{C} / \mathrm{T}$ ratio and the SUVmax value may provide valuable information about tumor differentiation in small adenocarcinomas.

Next, we classified adenocarcinomas into solid type and subsolid type. The solid type tumors all have a $\mathrm{C} / \mathrm{T}$ ratio of $100 \%$, consisting only of consolidation, and therefore the $\mathrm{C} / \mathrm{T}$ ratio cannot be used to predict invasiveness in this group. SUVmax is a significant independent variable for invasiveness in patients with solid type tumors, but only the $\mathrm{C} / \mathrm{T}$ ratio is independently correlated with invasiveness in those with subsolid type tumors. We suggest, based on these results, that the $\mathrm{C} / \mathrm{T}$ ratio should be evaluated first, and SUVmax should be used to predict invasiveness and prognosis only in patients with solid type tumors.

Finally, we evaluated suitable cutoff values for predicting invasiveness in both the solid- and subsolid types of

Table 5 Comparisons between non-invasive and invasive adenocarcinomas

\begin{tabular}{llll}
\hline & Non-invasive $(\mathbf{n = 8 6 )}$ & Invasive $(\mathbf{n}=\mathbf{7 7})$ & $\mathbf{p}$-value \\
\hline Age (years) & $65 \pm 8$ & $67 \pm 8$ & 0.184 \\
Body weight $(\mathbf{k g})$ & $56.8 \pm 10.7$ & $57.4 \pm 10.3$ & 0.704 \\
Blood sugar $(\mathbf{m g} / \mathbf{d L})$ & $95.7 \pm 14.9$ & $91.0 \pm 12.5$ & 0.031 \\
Size $(\mathbf{m m})$ & $17.8 \pm 6.7$ & $20.0 \pm 6.0$ & 0.016 \\
SUVmax & $1.86 \pm 1.80$ & $3.80 \pm 3.10$ & $<0.001$ \\
C/T ratio (\%) & $65.1 \pm 37.7$ & $88.9 \pm 22.0$ & $<0.001$ \\
\hline
\end{tabular}

SUVmax: maximum standardized uptake value; $\mathrm{C} / \mathrm{T}$ ratio: consolidation diameter/tumor diameter ratio. Values are means \pm standard deviations. 
Table 6 Postoperative outcomes with solitary lung adenocarcinomas

\begin{tabular}{lllll}
\hline & Total $(\mathbf{n})$ & Recurrence within 3 years $(\mathbf{n})$ & Recurrence rate & (\%) \\
\hline Invasive & & 61 & 15 & 25 \\
& Lobectomy & 54 & 14 & 26 \\
Non-invasive & Limited resection & 7 & 1 & 14 \\
& & 64 & 4 & 6 \\
& Lobectomy & 48 & 0 & 8 \\
\hline
\end{tabular}

tumors. The SUVmax cutoff is 4.4 in patients with solid type tumors. SUVmax is correlated with both tumor size and histopathologic characteristics, with well-differentiated adenocarcinomas demonstrating low SUVmax values. Thus, a SUVmax of < 4.4 may be helpful in determining that a solid type tumor is well-differentiated and noninvasive. However, the sensitivity for tumor invasiveness is only $55 \%$ at this cutoff value, meaning that PET/CT cannot absolutely exclude invasiveness. Therefore, an additional diagnostic method is necessary to increase the accuracy of this evaluation. It is important to note that adenocarcinomas with SUVmax values of $\geq 4.4$ are so highly invasive that they are unsuitable for limited resection. In the study of Ohtsuka et al., patients with Stage I lung adenocarcinomas showing an SUVmax of $\geq 3.3$ had poorer disease-free survival than those with an SUVmax <3.3, for patients with Stage I [14]. Turning our attention to subsolid tumors, the $\mathrm{C} / \mathrm{T}$ ratio cutoff was found to be $53 \%$. This is consistent with previous studies concluding that a $\mathrm{C} / \mathrm{T}$ ratio $<50 \%$ is consistent with a non-invasive tumor $[4,6,7]$. As the sensitivity for tumor invasiveness is $89 \%$ at this cutoff, high $\mathrm{C} / \mathrm{T}$ ratio tumors are unsuitable for limited resection.

This study has 3 limitations, the first of which is its retrospective and single-center nature. Second, we did not investigate histopathologic types other than adenocarcinoma; squamous cell carcinoma and large cell carcinoma are generally solid on TSCT and have a higher SUVmax than adenocarcinoma on PET/CT. Third, the PET/CT scans were acquired 50 minutes after the injection of FDG according to the protocol of our institution. This is somewhat early phase and may have influence on the value of SUVmax.

\section{Conclusions}

In conclusion, both the $\mathrm{C} / \mathrm{T}$ ratio on $\mathrm{TSCT}$ and the SUVmax value on PET/CT are significantly associated with the level of invasiveness in small adenocarcinomas. These measures are superior to tumor size for predicting tumor invasiveness. However, there is a difference between the 2 evaluations: SUVmax and C/T ratio were significantly associated with invasiveness of solid type adenocarcinoma and subsolid type adenocarcinoma, respectively. Thus, the $\mathrm{C} / \mathrm{T}$ ratio should be evaluated first, and then SUVmax should be used to predict invasiveness only in patients with solid type tumors.

\section{Abbreviations}

TSCT: Thin-section computed tomography; $C / T$ ratio: Ratio of the maximum diameter of consolidation to the maximum tumor diameter; FDG: ${ }^{18}$ F-fluorodeoxyglucose; PET/CT: Positron emission tomography/ computed tomography; SUVmax: Maximum standardized uptake value; OSEM: Ordered-subset expectation maximization; ROC: Receiver operating characteristic; AUC: Area under the curve; Cl: Confidential interval.

\section{Competing interests}

The authors declare that they have no competing interests.

\section{Authors' contributions}

SIw are responsible for the concept and design of the study. SIw drafted and wrote the paper. MK participated in the design of the study and helped to draft manuscript. MK, SIt, KK, and RI is contributed to the clinical data acquisition and reviewed the manuscript. SN contributed to critical revision of the manuscript. All authors read and approved the final manuscript.

\section{Acknowledgements}

We thank an experienced medical editor in JAM Post Inc. for English check and revision.

\section{Author details}

${ }^{1}$ Nagoya University Graduate School of Medicine, Department of Radiology, 65 Tsurumai-cho, Showa-ku, Nagoya 466-8550, Japan. ²Nagoya University Graduate School of Medicine, Department of Radiological and Medical Laboratory Sciences, 1-1-20 Daiko-Minami, Higashi-ku, Nagoya 461-8673, Japan.

Received: 12 November 2013 Accepted: 9 January 2014

Published: 22 April 2014

\section{References}

1. Suzuki K, Koike T, Asakawa T, Kusumoto M, Asamura H, Nagai K, Tada H, Mitsudomi T, Tsuboi M, Shibata T, Fukuda H, Kato H: A prospective radiological study of thin-section computed tomography to predict pathological noninvasiveness in peripheral clinical IA lung cancer (Japan Clinical Oncology Group 0201). J Thorac Oncol 2011, 6:751-756.

2. Koike T, Koike T, Yamato Y, Yoshiya K, Toyabe S: Prognostic predictors in non-small cell lung cancer patients undergoing intentional segmentectomy. Ann Thorac Surg 2012, 93:1788-1794.

3. Okada M, Nakayama H, Okumura S, Daisaki H, Adachi S, Yoshimura M, Miyata Y: Multicenter analysis of high-resolution computed tomography and positron emission tomography/computed tomography findings to choose therapeutic strategies for clinical stage IA lung adenocarcinoma. J Thorac Cardiovasc Surg 2011, 141:1384-1391.

4. Asamura H, Hishida T, Suzuki K, Koike T, Nakamura K, Kusumoto M, Nagai K, Tada H, Mitsudomi T, Tsuboi M, Shibata T, Fukuda H: Radiographically determined noninvasive adenocarcinoma of the lung: Survival outcomes of Japan Clinical Oncology Group 0201. J Thorac Cardiovasc Surg 2013, 146:24-30.

5. Matsunaga T, Suzuki K, Hattori A, Fukui M, Kitamura Y, Miyasaka Y, Takamochi K, Oh S: Lung cancer with scattered consolidation: detection of new independent radiological category of peripheral lung cancer on thinsection computed tomography. Interact Cardiovasc Thorac Surg 2013, 16:445-449. 
6. Aoki T, Tomoda Y, Watanabe H, Nakata H, Kasai T, Hashimoto H, Kodate M, Osaki T, Yasumoto K: Peripheral lung adenocarcinoma: correlation of thin-section $C T$ findings with histologic prognostic factors and survival. Radiology 2001, 220:803-809.

7. Ohde Y, Nagai K, Yoshida J, Nishimura M, Takahashi K, Suzuki K, Takamochi K, Yokose T, Nishiwaki Y: The proportion of consolidation to ground-glass opacity on high resolution $\mathrm{CT}$ is a good predictor for distinguishing the population of non-invasive peripheral adenocarcinoma. Lung Cancer 2003, 42:303-310

8. Suzawa N, Ito M, Qiao S, Uchida K, Takao M, Yamada T, Takeda K, Murashima S: Assessment of factors influencing FDG uptake in non-small cell lung cancer on PET/CT by investigating histological differences in expression of glucose transporters 1 and 3 and tumour size. Lung Cancer 2011, 72:191-198.

9. Vesselle H, Salskov A, Turcotte E, Wiens L, Schmidt R, Jordan CD, Vallieres E, Wood DE: Relationship between non-small cell lung cancer FDG uptake at PET, tumor histology, and Ki-67 proliferation index. J Thorac Oncol 2008, 3:971-978

10. Iwano S, Ito S, Tsuchiya K, Kato K, Naganawa S: What causes false-negative PET findings for solid-type lung cancer? Lung Cancer 2013, 79:132-136.

11. Grgic A, Yuksel Y, Groschel A, Schafers HJ, Sybrecht GW, Kirsch CM, Hellwig $D$ : Risk stratification of solitary pulmonary nodules by means of PET using (18)F-fluorodeoxyglucose and SUV quantification. Eur J NuCl Med Mol Imaging 2010, 37:1087-1094.

12. Murakami S, Saito H, Sakuma Y, Kondo T, Oshita F, Ito H, Tsuboi M, Hasegawa C, Yokose T, Kameda Y, Nakayama H, Yamada K: Prognostic value of preoperative FDG-PET in stage IA lung adenocarcinoma. Eur J Radiol 2012, 81:1891-1895.

13. Miyasaka $Y$, Suzuki K, Takamochi K, Matsunaga T, Oh S: The maximum standardized uptake value of fluorodeoxyglucose positron emission tomography of the primary tumour is a good predictor of pathological nodal involvement in clinical N0 non-small-cell lung cancer. Eur J Cardiothorac Surg 2013, 44:83-87.

14. Ohtsuka T, Nomori H, Watanabe K, Kaji M, Naruke T, Suemasu K, Uno K: Prognostic significance of [(18)F]fluorodeoxyglucose uptake on positron emission tomography in patients with pathologic stage I lung adenocarcinoma. Cancer 2006, 107:2468-2473.

15. Lu P, Yu L, Li Y, Sun Y: A correlation study between maximum standardized uptake values and pathology and clinical staging in nonsmall cell lung cancer. Nucl Med Commun 2010, 31:646-651.

16. Li M, Sun Y, Liu Y, Han A, Zhao S, Ma L, Zheng J, Yu J: Relationship between primary lesion FDG uptake and clinical stage at PET-CT for non-small cell lung cancer patients: An observation. Lung Cancer 2010, 68:394-397.

17. Ishibashi T, Kaji M, Kato T, Ishikawa K, Kadoya M, Tamaki N: 18 F-FDG uptake in primary lung cancer as a predictor of intratumoral vessel invasion. Ann Nucl Med 2011, 25:547-553.

18. Higashi K, Ito K, Hiramatsu Y, Ishikawa T, Sakuma T, Matsunari I, Kuga G, Miura K, Higuchi T, Tonami H, Yamamoto I: 18 F-FDG uptake by primary tumor as a predictor of intratumoral lymphatic vessel invasion and lymph node involvement in non-small cell lung cancer: analysis of a multicenter study. J Nucl Med 2005, 46:267-273.

19. Pechet TT, Carr SR, Collins JE, Cohn HE, Farber JL: Arterial invasion predicts early mortality in stage I non-small cell lung cancer. Ann Thorac Surg 2004, 78:1748-1753.

20. Iwano S, Koike W, Matsuo K, Kitano M, Kawakami K, Okada T, Naganawa S: Correlation between dynamic CT findings and pathological prognostic factors of small lung adenocarcinoma. Cancer Imaging 2012, 12:187-193.

21. Perkins NJ, Schisterman EF: The inconsistency of "optimal" cutpoints obtained using two criteria based on the receiver operating characteristic curve. Am J Epidemiol 2006, 163:670-675.

22. Nomori H, Mori T, Ikeda K, Yoshimoto K, lyama K, Suzuki M: Segmentectomy for selected cT1N0M0 non-small cell lung cancer: a prospective study at a single institute. J Thorac Cardiovasc Surg 2012, 144:87-93.
23. El-Sherif A, Gooding WE, Santos R, Pettiford B, Ferson PF, Fernando HC, Urda SJ, Luketich JD, Landreneau RJ: Outcomes of sublobar resection versus lobectomy for stage I non-small cell lung cancer: a 13-year analysis. Ann Thorac Surg 2006, 82:408-415.

24. Sienel W, Stremmel C, Kirschbaum A, Hinterberger L, Stoelben E, Hasse J, Passlick B: Frequency of local recurrence following segmentectomy of stage IA non-small cell lung cancer is influenced by segment localisation and width of resection margins-implications for patient selection for segmentectomy. Eur J Cardiothorac Surg 2007, 31:522-527.

doi:10.1186/1470-7330-14-3

Cite this article as: Iwano et al:: Prediction of pathologic prognostic factors in patients with lung adenocarcinomas: comparison of thin-section computed tomography and positron emission tomography/computed tomography. Cancer Imaging 2014 14:3.

\section{Submit your next manuscript to BioMed Central and take full advantage of:}

- Convenient online submission

- Thorough peer review

- No space constraints or color figure charges

- Immediate publication on acceptance

- Inclusion in PubMed, CAS, Scopus and Google Scholar

- Research which is freely available for redistribution

Submit your manuscript at www.biomedcentral.com/submit
C Biomed Central 